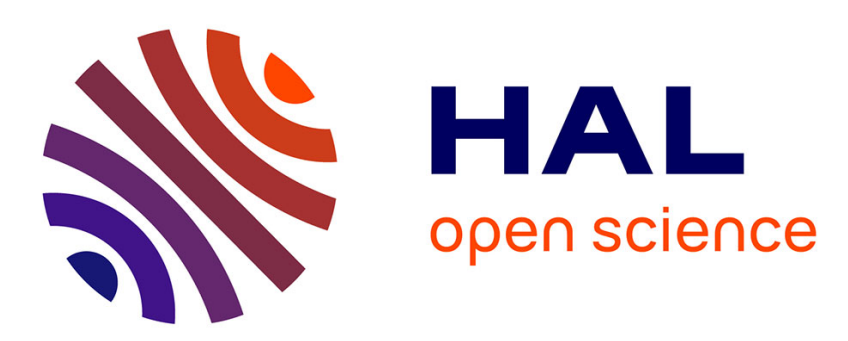

\title{
Restauration de la fertilité pour l'androstérilité génocytoplasmique chez le colza (Brassica napus L.). Utilisation des Raphano-Brassica
}

Patrick Rousselle, Françoise Dosba, Frédérique Eber

\section{To cite this version:}

Patrick Rousselle, Françoise Dosba, Frédérique Eber. Restauration de la fertilité pour l'androstérilité génocytoplasmique chez le colza (Brassica napus L.). Utilisation des Raphano-Brassica. Agronomie, 1986, 5 (5), pp.431-437. hal-00884828

\section{HAL Id: hal-00884828 \\ https://hal.science/hal-00884828}

Submitted on 1 Jan 1986

HAL is a multi-disciplinary open access archive for the deposit and dissemination of scientific research documents, whether they are published or not. The documents may come from teaching and research institutions in France or abroad, or from public or private research centers.
L'archive ouverte pluridisciplinaire HAL, est destinée au dépôt et à la diffusion de documents scientifiques de niveau recherche, publiés ou non, émanant des établissements d'enseignement et de recherche français ou étrangers, des laboratoires publics ou privés. 


\title{
Restauration de la fertilité pour l'androsté- rilité génocytoplasmique chez le colza (Brassica napus L.). Utilisation des Raphano-Brassica
}

\author{
Patrick ROUSSELLE ( $\left.{ }^{1}\right) \&$ Françoise DOSBA $\left({ }^{2}\right)$ \\ avec la collaboration technique de Frédérique EBER \\ I.N.R.A., Station d'Amélioration des Plantes, \\ Centre de Recherches de Rennes, B.P. 29, F 35650 Le Rheu
}

RÉSUMÉ

\begin{abstract}
Chez le colza, l'absence de restauration de la stérilité mâle génocytoplasmique d'origine radis conduit à envisager l'introgression de ce caractère, du radis au colza. La $1^{\text {re }}$ génération, issue du croisement entre le colza androstérile et un Raphano-Brassica (amphiploïde radis-chou) présente une bonne fertilité mâle. La présence de gènes de restauration est confirmée au cours des générations successives.

Les analyses de la méiose mâle de la $2^{e}$ génération montrent un doublement partiel du stock chromosomique. Ce phénomène est probablement lié à une fécondité femelle réduite.

Au cours des $3^{e}$ et $4^{e}$ générations, il apparaît quelques familles plus fécondes et une baisse sensible du nombre de chromosomes. L'obtention en $6^{\mathrm{e}}$ génération de plantes très fécondes doit permettre d'améliorer la restauration.
\end{abstract}

Mots clés additionnels : Fécondité femelle, fertilité pollinique, analyse cytogénétique, évolution chromosomique. (Raphanus sativus L.) to rape (Brassica napus L.)

\begin{abstract}
Rape lacks the restorer for cytoplasmic male sterility which is present in radish and this has led us to propose introduction of this character from radish to rape. The first generation from the cross between a male-sterile line of rape and a Raphano-Brassica (radish-kale amphiploid) had good male fertility. The presence of restorer genes was confirmed in the following generations. Analysis of male meiosis in the $2^{\text {nd }}$ generation showed incomplete doubling of the chromosome set. This was probably linked with the reduction of female fecundity. During the $3^{\text {rd }}$ and $4^{\text {th }}$ generations, some families appeared with better fecundity and a marked fall in chromosome number. The production of very prolific plants in the $6^{\text {th }}$ generation should allow further improvement of restoring ability.
\end{abstract}

Additional key words : Female fecundity, male fertility, cytogenetic analysis, chromosome evolution.

\section{INTRODUCTION}

L'androstérilité cytoplasmique découverte par OGURA (1968) chez le radis (Raphanus sativus L., $2 \mathrm{n}=18$, génome $\mathrm{R}$ ) ainsi que la fréquence élevée du (ou des) gène(s) de restauration (95 p. 100 des génoty-

\footnotetext{
Adresses actuelles :
}

(1) I.N.R.A., Station d'Amélioration de la pomme de terre et des plantes à bulbes, Domaine de Keraiber, Ploudaniel, F 29260 Lesneven.

(2) I.N.R.A., Station d'Arboriculture fruitière, Centre de Recherches de Bordeaux, F 33140 Pont-de-la-Maye. pes selon cet auteur) ont permis la création de variétés hybrides de radis (BONNET, 1975). Devant l'absence de stérilité mâle utilisable chez le chou (Brassica oleracea L., $2 \mathrm{n}=18$, génome C), BANNEROT et al. (1974) ont introduit par rétrocroisements successifs le cytoplasme mâle-stérile du radis chez cette espèce. Parmi les graines récoltées en fécondation non contrôlée de ce matériel, l'une d'elles a donné une plante mâlestérile de type colza. Par l'analyse de la méiose (CAUDERON, comm. pers., 1978), il est apparu que cette plante présentait un comportement asyndétique avec 37 chromosomes; une structure génomique de type 
ACR est donc fortement probable. Cette plante a servi de base à l'élaboration d'un programme de sélection pour la stérilité mâle chez le colza (Brassica napus L., $2 \mathrm{n}=38$, génomes $\mathrm{AC}$ ).

Dès les premières générations de sélection chez le colza, 2 inconvénients majeurs sont apparus: l'absence de restauration de la fertilité (RouSSELLE, 1982) et une importante déficience chlorophyllienne comme chez le chou (BANNEROT et al., 1977). L'utilisation de la fusion de protoplastes a résolu les problèmes de déficience chlorophyllienne (PELLETIER et al., 1983).

Malgré une prospection importante au sein de l'espèce $B$. napus, aucune solution n'a pu être apportée à la restauration de la fertilité. Nous avons alors supposé que le (ou les) gène(s) de restauration présent(s) chez le radis existai(en)t aussi chez les 2 amphiploïdes Raphano-Brassica (amphiploïde radis $\times$ chou, $2 \mathrm{n}=36$, génomes RC) obtenus par CHOPINET (1944) et par MAC NAUGHTON (1973). C'est pourquoi un programme de croisements interspécifiques faisant intervenir ces 2 Raphano-Brassica et une lignée mâle stérile de colza a été engagé pour transférer ce(s) gène(s) chez le colza et obtenir ainsi une restauration au niveau hétérozygote.

\section{MATÉRIEL}

Le géniteur de colza utilisé (S7) est mâle stérile. Il a été obtenu à l'issue du $4^{\mathrm{e}}$ rétrocroisement par la variété «Jet neuf » sur la plante mâle stérile de type colza ; la généalogie est décrite par RoUSSELLE (1982). Mise à part une déficience chlorophyllienne importante, ce géniteur a le phénotype colza avec notamment des pétales jaunes.

Le Raphano-Brassica obtenu par CHOPINET (RBC) a un port voisin de celui du radis mais ne tubérise pas. Les feuilles sont petites, découpées et pubescentes. Les pétales sont blancs et assez petits. Le fruit est une silique à bec comme chez le radis et l'autofertilité est largement préférentielle.

Le Raphano-Brassica reçu de MAC NAUGHTON (RBMN) a été sélectionné pour une utilisation fourragère. Il a un développement exubérant et des feuilles nombreuses, grandes et assez découpées. Les fleurs sont caractérisées par leur grande taille et les pétales blancs présentent des nervures jaunes ou violettes. Le fruit est une silique à bec et l'autofertilité est préférentielle.

\section{MÉTHODES}

Le schéma initial de transfert était construit sur le principe de croisements entre $\mathrm{S} 7$ et les RaphanoBrassica suivis de rétrocroisements systématiques par le colza et d'intercroisements entre les plus fertiles. Cependant, les difficultés liées à la production de graines nous ont contraints à utiliser également les fécondations libres.
L'appréciation de la déficience chlorophyllienne se fait par observation des plantules après vernalisation (4 semaines à $6{ }^{\circ} \mathrm{C}, 8 \mathrm{~h}$ de jour).

Les méioses sont observées en métaphase I des cellules mères du pollen selon la technique utilisée chez le blé (DOSBA \& DOUSSINAULT, 1978).

L'analyse de la structure chromosomique des différents géniteurs les uns par rapport aux autres, est effectuée selon la méthode utilisée chez les Triticinées (BOURGEOIS et al., 1978). Le nombre de translocations réciproques par lesquelles les 2 parents diffèrent est apprécié par le nombre de trivalents ou quadrivalents observés chez l'hybride. Le pourcentage de cellules présentant un ou plusieurs multivalents indique l'importance relative des translocations.

La culture in vitro d'embryons immatures sur milieu de MONNIER (1974) et la détermination de la viabilité du pollen sont réalisées selon les méthodes décrites précédemment (RoUSSELLE \& EBER, 1983). Le pourcentage de grains de pollen colorés en rouge au carmin acétique de BELLING donne un indice de la fertilité (If).

\section{RÉSULTATS. DISCUSSION}

\section{A. Première génération (G1)}

\section{Obtention et description des hybrides $57 \times$ Raphano-Brassica}

\section{a) Avec le Raphano-Brassica d'origine «CHOPI- $N E T \gg(R B C)$}

La culture in vitro d'embryons immatures a permis l'obtention de l'hybride entre S7 et RBC. 77 plantes ont été obtenues à partir de 507 embryons. Après vernalisation, ces jeunes plantes ne présentent pas de déficience chlorophyllienne. Leur allure générale en végétation est très voisine de celle du parent mâle (RBC) avec toutefois une pilosité foliaire moindre.

Les fleurs sont blanches et de même taille que celles du parent mâle. Cependant, dans de nombreux cas et particulièrement en fin de floraison, nous avons observé quelques fleurs isolées à secteurs jaunes ou plus rarement une ramification complète à fleurs jaunes. Dans de tels croisements, le caractère fleur blanche est dominant sur le caractère fleur jaune et peut être attribué à un chromosome venant du radis (FUKUSHIMA, 1945 ; SERNYK \& STEFANSSON, 1982). Les secteurs jaunes peuvent être dus à une perte locale du chromosome portant le caractère blanc.

\section{b) Avec le Raphano-Brassica d'origine «MAC NAUGHTON 》 (RBMN)}

8 plantes ont été obtenues par culture in vitro d'embryons immatures provenant du croisement entre S7 $\times$ RBMN. Aucune des plantes ne présente de déficience chlorophyllienne. Comme leur parent mâle, ces plantes sont très exubérantes. Les fleurs sont semblables à celles du parent mâle et présentent de larges pétales blancs nervurés de jaune ou de violet. 


\section{Etude de la restauration de la fertilité pollinique et de la fécondité}

Du fait que le parent femelle utilisé dans les croisements est une lignée mâle-stérile (S7), l'analyse de la capacité à la restauration des 2 Raphano-Brassica se fait par l'interprétation directe de l'indice de fertilité (If) des croisements S7 $\times$ RBC et S7 $\times$ RBMN.

\section{a) Croisement $S 7 \times R B C$}

If varie de 3 à 82 p. 100 avec une moyenne de 46,4 p. 100 . Nous pouvons donc admettre que cet hybride Raphano-Brassica permet la restauration de la fertilité du colza mâle-stérile S7. HEYN (1976) a remarqué la présence de pollen chez l'hybride entre une lignée femelle de même origine que S7 et RBC. Il a conclu que $\mathrm{RBC}$ est un restaurateur potentiel. La grande variabilité des If doit être due aux différences d'expression des gènes en cause suivant les plantes et à une stérilité de type chromosomique chez cet hybride interspécifique.

En dépit d'une assez bonne fertilité mâle, la fécondité du matériel appréciée par le nombre de descendants est faible : 126 graines ont été obtenues pour l'ensemble des 77 plantes. La presque totalité (108) de ces graines a été obtenue par croisement frère-sœur, les autres en autofécondation (12), ou en croisement avec du colza (6). Sur 77 plantes de $1^{\text {re }}$ génération, 37 n'ont pas eu de descendance et 5 plantes seulement ont été représentées dans la $2^{e}$ génération par plus de 5 plantes.

Il n'y a pas de corrélation significative entre la fertilité pollinique, d'une part, et le nombre de graines par plante, d'autre part. Une analyse par classe du nombre de graines (tabl. 1) montre une augmentation de la moyenne de la fertilité pollinique, mais ces moyennes ne sont pas significativement différentes. Les 2 résultats, bien que non significatifs statistiquement, peuvent permettre d'envisager une même tendance pour ces 2 phénomènes : fertilité pollinique et fécondité.

\section{b) Croisement $S 7 \times R B M N$}

Parmi les 8 plantes obtenues, 7 sont stériles. La dernière présente de faibles traces de pollen. Nous pouvons donc admettre qu'il n'a pas été possible de mettre en évidence des gènes nécessaires à la restauration chez RBMN. Ces observations sont en accord avec celles de HEYN (1978) lorsqu'il utilise ce géniteur en

\section{TABLEAU}

Fertilité moyenne des plantes de lre génération issues du croisement $S 7 \times R B C$ par classe de fécondité.

Medium fertility of first generation plants from the cross $S 7 \times R B C$ for each fecundity class.

\begin{tabular}{llll}
\hline Classes de fécondité (1) & 0 & 1 et 2 & 3 et + \\
\hline Nombre de plantes & 15 & 11 & 14 \\
\hline Fertilité moyenne & 35,33 & 44,85 & 47,57 \\
\hline Ecart type & 11,95 & 10,71 & 11,08 \\
\hline \hline
\end{tabular}

(1) Appréciée par le nombre de graines obtenues par silique. Estimated as seed number per pod. croisement comme mâle avec un colza mâle stérile. Il faut cependant noter le faible nombre de plantes issues de ce croisement en $1^{\text {re }}$ génération. De plus, aucune descendance n'a pu être obtenue.

\section{Analyse cytologique}

\section{a) Hybrides $S 7 \times R B C$}

Le comportement méiotique des plantes hybrides diffère de celui des 2 parents (tabl. 2). Celui des parents est régulier, présente un faible taux d'asyndèse et peu de multivalents par cellule. Chez les plantes hybrides, pour lesquelles le nombre euploïde de chromosomes est 37 , on remarque :

- des plantes aneuploïdes ( $4 / 24$ observées) ayant 33,38 et 42 chromosomes, issues vraisemblablement de gamètes parentaux déséquilibrés ;

- un nombre moyen d'univalents par cellule élevé et dû principalement au manque d'appariement de chromosomes des génomes $\mathrm{A}$ et $\mathrm{R}$; il est toutefois inférieur à 19, nombre théoriquement attendu $(10 \mathrm{~A}+9 \mathrm{R})$;

- un nombre moyen de bivalents par cellule plus élevé que celui correspondant à l'association des chromosomes des 2 génomes $\mathrm{C}$ mis en présence ( 9 bivalents prévisibles) ;

- la présence fréquente d'un ou plusieurs multivalents par cellule ;

- un taux d'asyndèse n'atteignant pas 30 p. 100 alors qu'il devrait atteindre 51 p. 100 .

\section{b) Hybrides $S 7 \times R B M N$}

Le comportement méiotique de RBMN est régulier avec toutefois un taux d'asyndèse plus élevé que celui de RBC (tabl. 2). Chez les hybrides S7 $\times$ RBMN, 2 plantes sur les 5 analysées sont aneuploïdes $(2 n=35$ ou 36 chromosomes). La perte d' 1 ou 2 chromosomes se traduit par une diminution du nombre d'univalents qui proviennent plutôt des génomes $\mathrm{R}$ ou $\mathrm{A}$ que des génomes $\mathrm{C}$ censés s'apparier de manière préférentielle. Chez les 3 plantes euploïdes, le nombre moyen d'univalents par cellule est assez faible et n'atteint jamais les 19 univalents attendus (tabl. 2). De plus, de nombreuses cellules présentent des multivalents.

\section{c) Comparaison des hybrides $S 7 \times R B C$ et $S 7 \times R B M N$}

En dépit du faible nombre de cellules observées par plante, le comportement des plantes à l'intérieur d'un croisement est suffisamment homogène pour permettre une interprétation globale des résultats. Si les génomes $\mathrm{R}$ et $\mathrm{A}$ ne sont pas homéologues et si la régulation génétique de l'appariement des chromosomes aboutit comme chez les parents à un comportement de type diploïde, les 2 génomes $A$ et $R$ ne devraient pas s'associer. Malgré un taux d'asyndèse plus élevé chez S7 $\times$ RBMN (sans doute lié au taux d'asyndèse de RBMN) que chez S7 $\times$ RBC, seules 42 cellules sur 575 (réparties uniquement dans 2 plantes) présentent au moins les 19 univalents attendus. De plus, chez 1 plante de l'hybride $\mathrm{S} 7 \times \mathrm{RBC}$, le nombre de chromosomes sous forme d'univalents n'excède pas 6 et peut être nul. Ceci signifie que tout ou partie des 
TABLEAU 2

Comportement méiotique des hybrides $S 7 \times R B C$ et $S 7 \times R B M N$ et leurs parents. Meiotic behaviour of $S 7 \times R B C$ and $S 7 \times R B M N$ hybrids and their parents.

\begin{tabular}{|c|c|c|c|c|c|c|c|c|c|c|c|}
\hline Généalogie & Génomes & $2 n$ & $\begin{array}{l}\text { Nombre de } \\
\text { plantes }\end{array}$ & $\begin{array}{l}\text { Nombre de } \\
\text { cellules }\end{array}$ & & I & II & III & IV & Autres & $\begin{array}{c}\% \text { chromosomes } \\
\text { univalents }\end{array}$ \\
\hline S7 & $\mathrm{AACC}$ & 38 & 2 & 86 & $\begin{array}{l}\text { (1) } \\
\text { (2) }\end{array}$ & $\begin{array}{l}0,40 \\
0-2\end{array}$ & $\begin{array}{l}18,76 \\
16-19\end{array}$ & & $\begin{array}{l}0,02 \\
0-1\end{array}$ & & 1,0 \\
\hline $\mathrm{RBC}$ & RRCC & 36 & 1 & 80 & & $\begin{array}{l}0,20 \\
0-2\end{array}$ & $\begin{array}{l}17,90 \\
17-18\end{array}$ & & & & 0,6 \\
\hline \multirow[t]{4}{*}{$\mathrm{S} 7 \times \mathrm{RBC}$} & $\mathrm{ARCC}$ & 37 & 20 & 359 & & $\begin{array}{r}10,94 \\
1-23\end{array}$ & $\begin{array}{r}10,65 \\
4-18\end{array}$ & $\begin{array}{l}0,98 \\
0-5\end{array}$ & $\begin{array}{l}0,43 \\
0-2\end{array}$ & $\begin{array}{l}0,02 \\
0-1\end{array}$ & 29,7 \\
\hline & & 38 & 2 & 20 & & $\begin{array}{l}7,15 \\
0-12\end{array}$ & $\begin{array}{r}12,45 \\
8-19\end{array}$ & $\begin{array}{l}0,45 \\
0-2\end{array}$ & $\begin{array}{l}1,15 \\
0-3\end{array}$ & & 13,6 \\
\hline & & 33 & 1 & 15 & & $\begin{array}{l}8,67 \\
4-11\end{array}$ & $\begin{array}{r}10,40 \\
8-12\end{array}$ & $\begin{array}{l}0,47 \\
0-1\end{array}$ & $\begin{array}{l}0,53 \\
0-2\end{array}$ & & 26,3 \\
\hline & & 42 & 1 & 19 & & $\begin{array}{l}9,05 \\
6-13\end{array}$ & $\begin{array}{l}14,90 \\
14-16\end{array}$ & $\begin{array}{l}1,05 \\
0-2\end{array}$ & & & 24,0 \\
\hline RBMN & RRCC & 36 & 1 & 62 & & $\begin{array}{l}1,12 \\
0-2\end{array}$ & $\begin{array}{l}17,44 \\
16-18\end{array}$ & & & & 3,1 \\
\hline \multirow[t]{3}{*}{$\mathrm{S} 7 \times \mathrm{RBMN}$} & ARCC & 37 & 3 & 116 & & $\begin{array}{r}13,19 \\
7-17\end{array}$ & $\begin{array}{l}9,93 \\
7-14\end{array}$ & $\begin{array}{l}0,89 \\
0-4\end{array}$ & $\begin{array}{l}0,32 \\
0-2\end{array}$ & & 35,7 \\
\hline & & 36 & 1 & 21 & & $\begin{array}{r}10,94 \\
7-16\end{array}$ & $\begin{array}{l}9,72 \\
7-14\end{array}$ & $\begin{array}{l}0,86 \\
0-2\end{array}$ & $\begin{array}{l}0,76 \\
0-2\end{array}$ & & 30,4 \\
\hline & & 35 & 1 & 22 & & $\begin{array}{r}11,32 \\
8-14\end{array}$ & $\begin{array}{l}9,31 \\
7-13\end{array}$ & $\begin{array}{l}1,14 \\
0-3\end{array}$ & $\begin{array}{l}0,41 \\
0-1\end{array}$ & & 32,3 \\
\hline
\end{tabular}

(1) Nombre moyen/cellule ;

(2) Extrêmes ; $\quad I$ = univalent ;

II = bivalent ;

III = trivalent ;

$\mathrm{IV}=$ quadrivalent

chromosomes du génome $\mathrm{R}$ peut s'associer avec les chromosomes des génomes $\mathrm{A}$ ou $\mathrm{C}$. L'information génétique du génome $R$ peut donc être introduite par recombinaison chez le colza.

L'excès de chromosomes associés sous forme de bivalents ou multivalents chez les 2 hybrides $\mathrm{S} 7 \times \mathrm{RBC}$ et $\mathrm{S} 7 \times \mathrm{RBMN}$ peut être expliqué de différentes façons.

Tout d'abord, une certaine homéologie doit exister entre les génomes mis en présence. Ainsi des appariements homéologues ont-ils déjà été mis en évidence chez des colzas haploïdes qui présentaient 39 à 100 p. 100 de chromosomes associés sous forme de bivalents ou de multivalents au lieu du très faible pourcentage attendu (RENARD \& DOSBA, 1980).

Par ailleurs, dans les hybrides radis $x$ navette (RA), TOKUMASU (1970) a observé une moyenne de
2,6 bivalents par cellule et, dans les croisements radis $\times$ colza (RAC), RouSSELLE (1983) a observé 8 bivalents. L'ensemble de ces analyses renforce les hypothèses d'homéologie entre les génomes $\mathrm{A}, \mathrm{R}$ et $\mathrm{C}$.

Les différences d'associations observées peuvent être dues aussi à des différences de structure chromosomique au niveau des génomes mis en présence. L'examen du tableau 3 fait apparaître une distinction entre $\mathrm{S} 7$ et $\mathrm{RBC}$ par au moins 3 translocations réciproques ; RBMN se distinguerait de la lignée de colza par 2 translocations. L'analyse des croisements réciproques entre $\mathrm{RBC}$ et RBMN met en évidence au moins 2 translocations réciproques qui différencient les 2 amphiploïdes. D'une manière générale, l'importance des translocations détectées est assez faible puisque le pourcentage de cellules avec un ou plusieurs multivalents n'excède pas 35 .

TABLEAU

Pourcentage de cellules présentant un ou plusieurs multivalents chez des hybrides avec $S 7, R B C$ ou $R B M N$. Percentage of cells with one or more multivalents in hybrids involving $S 7, R B C$ or RBMN.

\begin{tabular}{|c|c|c|c|c|c|c|c|c|c|c|}
\hline \multirow{2}{*}{ Généalogie } & \multirow{2}{*}{ Génome } & \multirow{2}{*}{$\begin{array}{l}\text { Nombre de } \\
\text { plantes }\end{array}$} & \multirow{2}{*}{$\begin{array}{l}\text { Nombre de } \\
\text { cellules }\end{array}$} & \multicolumn{7}{|c|}{ Pourcentage de cellules avec } \\
\hline & & & & 1 & III ou IV & 2 & III ou IV & 3 & III ou IV & + de 3 III ou IV \\
\hline S7 & $\mathrm{AACC}$ & 2 & 86 & & 2 & & & & & \\
\hline RBC & $\mathrm{RR} C \mathrm{C}$ & 1 & 80 & & 0,6 & & & & & \\
\hline $\mathrm{S} 7 \times \mathrm{RBC}$ & $\mathrm{AR} C \mathrm{C}$ & 20 & 359 & & 27 & & 26 & & 15 & 5 \\
\hline RBMN & RR CC & 1 & 62 & & & & & & & \\
\hline $\mathrm{S} 7 \times \mathrm{RBMN}$ & $\mathrm{AR} \mathrm{CC}$ & 3 & 116 & & 35 & & 30 & & 1 & 1 \\
\hline $\begin{array}{l}\mathrm{RBC} \times \mathrm{RBMN} \\
\text { et réciproque }\end{array}$ & RR CC & 4 & 142 & & 20 & & 28 & & 1 & \\
\hline
\end{tabular}

$\mathrm{III}=$ trivalent IV = quadrivalent 


\section{B. Générations G2, G3, G4 et G5 du croisement S7 $\times$ RBC}

\section{Obtention et description}

La faible fécondité est un inconvénient majeur pour l'obtention des différentes générations. Aucune plante n'est issue de croisement contrôlé ; chaque génération est issue de la précédente par intercroisement libre entre les plantes du fait de l'absence d'autres colzas en fleurs en même temps. Il s'agit donc essentiellement de croisements frère-sœur. Nous avons préconisé cette méthode dans un premier temps plutôt que le rétrocroisement systématique avec le colza (HEYN, 1976). Dans notre cas, il est vraisemblable que les possibilités de recombinaison entre le génome $R$ et les 2 génomes $\mathrm{A}$ et $\mathrm{C}$ sont plus importantes.

L'allure générale des plantes (port, feuillage et pilosité) est semblable à celle observée en $1^{\text {re }}$ génération. Les plantes ne présentent pas de déficience chlorophyllienne. D'après les observations réalisées pár d'autres auteurs (BANNEROT et al., 1977 ; MAC COLLUM, 1979 ; KATO \& TOKUMASU, 1980), la déficience apparaît lorsque le stock chromosomique de l'espèce donneuse du cytoplasme est en grande partie éliminé par rétrocroisement avec l'autre espèce. Dans le cas présent, comme l'étude cytologique le montrera, les plantes possèdent tout ou partie des génomes $\mathrm{R} d u$ radis et $A C$ du colza. Ainsi, la déficience chlorophyllienne ne peut être appréciée avant plusieurs générations.

La plupart des plantes ont des pétales blancs. Ce caractère qui provient du radis est dominant.

\section{Etude de la fertilité et de la fécondité}

La mesure de la fertilité par If n'a été effectuée que pour la génération G2. Pour les générations G3, G4 et G5, elle a été estimée visuellement sans comptage. La moyenne générale de If en $\mathrm{G} 2$ est de 55,2 , ce qui représente une nette amélioration par rapport à la génération G1. Une analyse de la variance à modèle hiérarchique (tabl. 4) a permis l'estimation de cette dernière pour les 3 niveaux (Intra-plante, Intra G2 et Inter G2). Malgré une forte variance intra-plante qui laisse supposer un échantillonnage limite, il est intéressant de remarquer que la variance intra G2 est plus de 8 fois supérieure à la variance inter G2. Il était attendu que la variabilité soit plus forte en G2 qu'en G1 et cela nous renforce dans l'idée de garder toutes les plantes en G2, ce qui était souhaitable compte tenu de l'origine interspécifique du matériel.

La fécondité, appréciée par le pourcentage de plantes ayant une descendance, diminue des générations G1 à G3. Le pourcentage augmente largement par la suite (tabl. 5). Le nombre élevé de chromosomes dû à la formation de gamètes non réduits peut être la cause principale de cette fécondité faible. Les 2 taux élevés observés en G4 et en G5 (77 et 91 p. 100 respectivement) sont dus à l'importance croissante d'une plante ( $\left.{ }^{\circ} 42\right)$ qui présente une fécondité supérieure à la moyenne en G1 et en G2. Par contre, son comportement cytologique est similaire à celui des autres plantes. En G6, seuls les descendants de cette plante restaurée subsistent et sont représentés par plus de 2400 individus.
TABLEAU 4

Analyse de la variance de la fertilité de la génération G2. Analysis of variance of fertility within the G2 generation.

\begin{tabular}{lcc}
\hline \hline Source de variation & Variances estimées & D.D.L. \\
\hline Intra plante & 64,7 & 219 \\
\hline Intra G2 & 88,7 & 42 \\
\hline $\begin{array}{l}\text { Inter G2 } \\
\text { Intra G1 }\end{array}$ & 10,8 & 29 \\
\hline \hline
\end{tabular}

$\mathrm{F}=\frac{\text { Intra } \mathrm{G} 2}{\text { Inter } \mathrm{G} 2}=8,21 \quad$ HS (seuil $=2,32$ ).

TABLEAU 5

Evolution de la fécondité au cours des différentes générations. Change in fecundity over successive generations.

\begin{tabular}{ccccc}
\hline \hline & Nombre & \multicolumn{2}{c}{ Pourcentage de plantes } & \\
\cline { 3 - 4 } Génération & $\begin{array}{c}\text { Notal } \\
\text { de plantes }\end{array}$ & $\begin{array}{c}\text { avec } \\
\text { descendance }\end{array}$ & $\begin{array}{c}\text { sans descendance } \\
\text { plante 42 }\end{array}$ & \\
\hline G1 & 77 & 52 & 48 & $1 / 77$ \\
G2 & 126 & 31 & 69 & $4 / 126$ \\
G3 & 146 & 4 & 96 & $3 / 146$ \\
G4 & 22 & 77 & 23 & $13 / 22$ \\
G5 & 33 & 91 & 9 & $30 / 33$ \\
\hline \hline
\end{tabular}

Cette fécondité réduite gêne l'efficacité de la sélection. Ainsi en G6, le sélectionneur a de nombreuses plantes qui présentent l'avantage d'une bonne fécondité, mais ce matériel provenant en entier de la plante 42 ne représente qu'une part réduite de la variabilité potentielle du croisement de départ alors qu'aucune sélection pour les caractères agronomiques n'a été réalisée.

\section{Analyse cytologique}

L'analyse de l'évolution chromosomique de la descendance du croisement $\mathrm{S} 7 \times \mathrm{RBC}$ révèle un accroissement important du nombre de chromosomes et une fluctuation notable entre plantes dès la $2^{\mathrm{e}}$ génération après le croisement (tabl. 6). Le nombre élevé d'associations chromosomiques à la génération $F_{1}$ (tabl. 2) a abouti à la formation de gamètes mâles et femelles partiellement (ou non) réduits. Ainsi, on approche la situation amphiploïde AA CC RR CC. Le faible pourcentage de chromosomes univalents pourrait correspondre aux chromosomes qui se retrouvent à l'état haploïde et le pourcentage de chromosomes sous forme de multivalents résulterait de l'état tétraploïde du génome $\mathrm{C}$ et des éventuelles translocations. Une réduction lente du nombre de chromosomes s'amorce au cours des $3^{\mathrm{e}}$ et $4^{\mathrm{e}}$ générations, accompagnée de l'augmentation du pourcentage de chromosomes univalents en $G_{4}$. Ce retour lent vers l'état euploïde permet une sélection des types " colza " et une fixation des gènes de restauration venant $d u$ génome $R$ du radis. 
TABLEAU 6

Analyse cytologique des générations G2, G3 et G4 issues du croisement $S 7 \times R B C$.

Cytological analysis of $G 2, G 3$ and $G 4$ generations from $S 7 \times R B C$ hybrid.

\begin{tabular}{|c|c|c|c|c|c|c|c|c|c|}
\hline \multirow{2}{*}{$\begin{array}{l}\text { Génération } \\
\text { après le } \\
\text { croisement }\end{array}$} & \multirow{2}{*}{ Généalogie } & \multirow{2}{*}{$\begin{array}{l}\text { Nombre de } \\
\text { plantes }\end{array}$} & \multirow{2}{*}{$\begin{array}{l}\text { Nombre de } \\
\text { cellules }\end{array}$} & \multirow{2}{*}{$2 n$} & \multicolumn{5}{|c|}{ Pourcentage de chromosomes sous forme } \\
\hline & & & & & 1 & II & III & IV & Autres \\
\hline \multirow{6}{*}{$\begin{array}{l}\mathrm{G} 2 \\
\mathrm{G} 2\end{array}$} & S7 & 2 & 86 & 38 & 0,8 & 98,5 & - & 0,7 & - \\
\hline & RBC & 1 & 80 & 36 & 0,6 & 99,4 & - & - & - \\
\hline & Crésor & 1 & 24 & 38 & - & 100 & - & - & - \\
\hline & $(\mathrm{S} 7 \times \mathrm{RBC})^{2}$ & 16 & 288 & 43 à 73 & 6,7 & 83,8 & 3,3 & 4,9 & 1,3 \\
\hline & $(\mathrm{S} 7 \times \mathrm{RBC})$ & & & & & & & & \\
\hline & $\begin{array}{c}\times \\
\text { Crésor }\end{array}$ & 1 & 32 & 44 & 22,7 & 70,9 & 1,9 & 4,5 & - \\
\hline G3 & $(\mathrm{S} 7 \times \mathrm{RBC})^{3}$ & 18 & 420 & 46 à 69 & 3,9 & 87,8 & 2,8 & 4,9 & 0,6 \\
\hline G4 & $(\mathrm{S} 7 \times \mathrm{RBC})^{4}$ & 13 & 109 & 34 à 67 & 17,9 & 68,3 & 8,0 & 5,1 & 0,7 \\
\hline
\end{tabular}

I = Univalent ; $\quad$ II = Bivalent ; $\quad$ III = Trivalent ; $\quad$ IV = Quadrivalent.

En procédant par rétrocroisements successifs, on retournerait plus rapidement vers le génotype colza, comme semble l'indiquer le croisement "Crésor " $X$ $(\mathrm{S} 7 \times \mathrm{RBC})$ figurant dans le tableau 6. Mais le risque de perdre l'information génétique venant du radis est alors grand.

\section{CONCLUSIONS. PERSPECTIVES}

Le Raphano-Brassica d'origine «CHOPINET》 (RBC) semble bien permettre la restauration de la fertilité en G1. Cela paraît moins évident pour le RaphanoBrassica d'origine « MAC NAUGHTON » (RBMN) avec cependant les restrictions suivantes : les effectifs obtenus avec RBMN sont faibles et des plantes peu fertiles sont aussi apparues avec RBC.
Le retour du matériel à un état stable du point de vue chromosomique est la phase la plus délicate du programme compte tenu du taux d'appariement faible entre les génomes en présence ; l'absence de rétrocroisements au cours des premières générations a permis de conserver la plus grande part du génome $\mathrm{R}$.

En réalité, c'est la fécondité du matériel qui a réalisé la sélection pour aboutir en $6^{\mathrm{e}}$ génération à la descendance de la seule plante $n^{\circ} 42$ qui est maintenant le géniteur de base. Dans la mesure où ce géniteur était suffisamment hétérozygote, il sera possible de réaliser une sélection agronomique efficace de lignées de colza restauratrices par rétrocroisement systématique avec des géniteurs du programme « variétés hybrides ».

Reçu le 23 mars 1984. Accepté le 31 décembre 1984.

\section{RÉFÉRENCES BIBLIOGRAPHIQUES}

Bannerot H., Boulidard L., Cauderon Y., Tempe J., 1974. Cytoplasmic male sterility transfer from Raphanus to Brassica. Proc. Eucarpia Dundee Crop Sect., 25, 52-54.

Bannerot H., Boulidard L., Chupeau Y., 1977. Unexpected difficulties with the radish cytoplasm in Brassica oleracea. Eucarpia Cruciferae Newsletter, $\mathrm{N}^{\circ}$ 2, Décembre 1977, 16.

Bonnet A., 1975. Introduction et utilisation d'une stérilité mâle cytoplasmique dans les variétés précoces européennes de radis (Raphanus sativus L.). Ann. Amélior. Plantes, 25, 381-397.

Bourgeois F., Dosba F., Douaire G., 1978. Analyse et identification des translocations réciproques présentes chez le géniteur V.P.M. et les variétés " Marne ", " Moisson » et " Roazon ». Ann. Amélior. Plantes, 28, 411-429.

Chopinet R., 1944. Hybrides intergénériques Raphano-Brassica. Rev. Hortic., 2111, 98-100.

Dosba F., Doussinault G., 1978. Création de lignées de blé présentant les caractéristiques agronomiques favorables d'Ae, ventricosa. Ann. Amélior. Plantes, 28, 27-44.
Fukushima E., 1945. Cytogenetic studies on Brassica and Raphanus. I Studies on the intergeneric F1 hybrids between Brassica and Raphanus. J. Dept. Agric. Kyushu Imp. Univ., 7, 281-400.

Heyn F. W., 1976. Transfer of restorer genes from Raphanus to cytoplasmic male-sterile Brassica napus. Cruciferae Newsletter, 1, 15.

Heyn F. W., 1978. Introgression of restorers genes from Raphanus sativus into cytoplasmic male sterile Brassica napus and the genetics of fertility restoration. Proc. Sth. Int. Conf. Rapeseed, Malmö, 8283.

Kato M., Tokumasu S., 1980. Nucleus substitution of Brassica japonica with Raphanus sativus and its resultant chlorophyll deficiency. Euphytica, 29, 97-106.

Mac Collum G. D., 1979. Sterility in successive back crosses of Raphano-Brassica with recurrent Brassica oleracea. Can. J. Genet. Cytol., 21, 479-485.

Mac Naughton I. H., 1973. Synthesis and sterility of RaphanoBrassica. Euphytica, 22, 70-88. 
Monnier M., 1974. Culture in vitro de l'embryon immature de Capsella bursa pastoris Moench. Thèse Doct. ès-Sci. Nat., Univ. Paris VI, $186 \mathrm{p}$.

Ogura H., 1968. Studies of the new male-sterility in japanese radish, with special reference to the utilization for this sterility towards the practical raising of hybrid seed. Mem. Fac. Agric. Kagoschima, 6, 39-78.

Pelletier G., Primard C., Vedel F., Chetrit P., Rémy R., Rousselle P., Renard M., 1983. Intergeneric cytoplasmic hybridization in Cruciferae by protoplast fusion. Mol. Gen. Genet., 191, 244-250.

Renard M., Dosba F., 1980. Etude de l'haploïdie chez le colza (Brassica napus L. var. oleifera Metzger). Ann. Amélior. Plantes, 30, 191-209.

Rousselle P., 1982. Premiers résultats d'un programme d'introduc- tion de l'androstérilité "Ogura " du radis chez le colza. Agronomie, 2, 859-864.

Rousselle P., 1983. Le croisement radis $\times$ colza. Réunion Groupe "Cytogénétique », Montpellier, 10/11 mai 1983.

Rousselle P., Eber F., 1983. Croisements interspécifiques entre quelques Brassica et Brassica napus L. Analyse génomique des hybrides et perspectives d'obtention de systèmes d'androstérilité chez le colza. Agronomie, 3, 153-159.

Sernyk J. L., Stefansson B. R., 1982. White flower color in rape associated with a radish chromosome. Can. J. Genet. Cytol., 24, 729-734.

Tokumasu S., 1970. Intergeneric hybrids between Brassica japonica and Raphanus sativus. Mem. Coll. Agric. Ehime Univ., 14, 1-18. 